\title{
Sensing Noise as an Information Object in Socio- Technical Environmental Monitoring
}

\author{
Sharon A Cox and Wenyan $\mathrm{Wu}$ \\ School of Computing and Digital Technology, Birmingham City University, Birmingham, UK \\ Sharon.cox@bcu.ac.uk
}

\begin{abstract}
A noise phenomenology is proposed that considers noise as an information object that only has meaning when experienced by an actor. Environmental noise has a major impact on the health and well-being of citizens, particularly when the noise occurs at night. Noise level monitoring can fail to take into account the impact of noise on the lived experienced of citizens. A socio-technical multi-sensor architecture is used to record night time noise in a domestic urban environment in a longitudinal study in the UK. Results are triangulated with contemporaneous logs of occupants to isolate noise patterns that cause the most disturbance to sleep behaviour. The phenomenology identifies the need for a multidimensional interdisciplinary approach to developing sensor networks to acquire noise information for environmental monitoring.
\end{abstract}

Keywords—phenomenology; noise levels; lived experience; environment monitoring; urban soundscape.

\section{INTRODUCTION}

Noise pollution in the nocturnal environment directly and indirectly affects physical and mental well-being of citizens [1]. The World Health Organization estimates that 903,000 years of life are lost each year due to noise-induced sleep disturbance for citizens of towns of $>50,000$ inhabitants in the European Union [2]. The impact of noise disturbance on sleep depends on the information content of the noise, the individual and the situation [3]. Previous phenomenological studies have focused on musical sounds rather than every day sounds [4]. We propose a noise phenomenology for unwanted environmental noise based on the document phenomenology of [5] and the intelligence and interoperability levels used by [6] that differentiates levels of meaning.

Fig. 1 presents a noise phenomenology that focuses on noise as an information object. An information object comprises of intrinsic and extrinsic information. Intrinsic information is the physical properties, diachronic attributes that stay the same across time and space [7]. Extrinsic information are attributed properties, contextual information about the purpose and role of the object in a wider system [5]. Meaning is derived from information objects by actors [8] and is influenced by, for example, an actor's personality traits, emotional state and past experience.

The information object can be considered at three levels: the object, the parts within it and the wider system within which it exists [5].

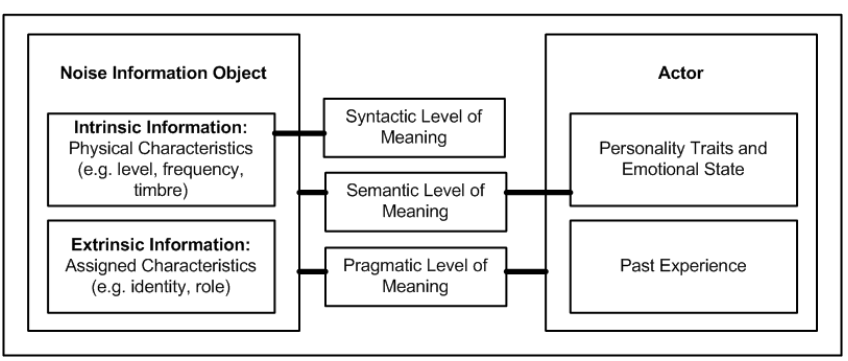

Fig. 1. Noise Phenomenology Noise Phenomenology based on [5] [6]

As an information object, noise can be decomposed into its intrinsic attributes such as level, frequency and tone that form syntactic information. The combination of the attributes are interpreted by an actor and assigned a semantic descriptor, such as loud or dull. The semantic information may be influenced by the personality traits and emotional state of the actor. Extrinsic information is attributed to the noise as it is identified and its role determined. Pragmatic information, information in context [6] is derived from combining what is known about the noise based on the wider context in which the noise is taking place and the actor's past experience. For example, the noise is an alarm and the building needs to be evacuated.

Environmental monitoring typically only focuses on the syntactic information; to fully understand the impact of noise, lived experience has to be explored. Phenomenological research aims to understand the lived experienced of the participants [9]. Lived experience is the sum of an individual's experiences [10] from interactions and transactions in the realworld. A socio-technical approach to environmental monitoring therefore needs a multi-sensor architecture to capture information about the noise object, the environment in which the noise occurs and the actor's response to the noise.

\section{EXPERIMENTAL SETUP}

Extrinsic information is based on noise identification. This is difficult due to a lack of annotated audio data in urban acoustic environments [11] and challenges of noise identification in real-world settings, such as overlapping sounds and false recognition. Sound recognition is only false when compared with other data [4]. A multi-sensor architecture was therefore used (Fig. 2) to capture a range of data about the environment in which the noise was recorded to assist with 
identifying and validating the identification of the noise based on the generic monitoring framework of [12] [13].

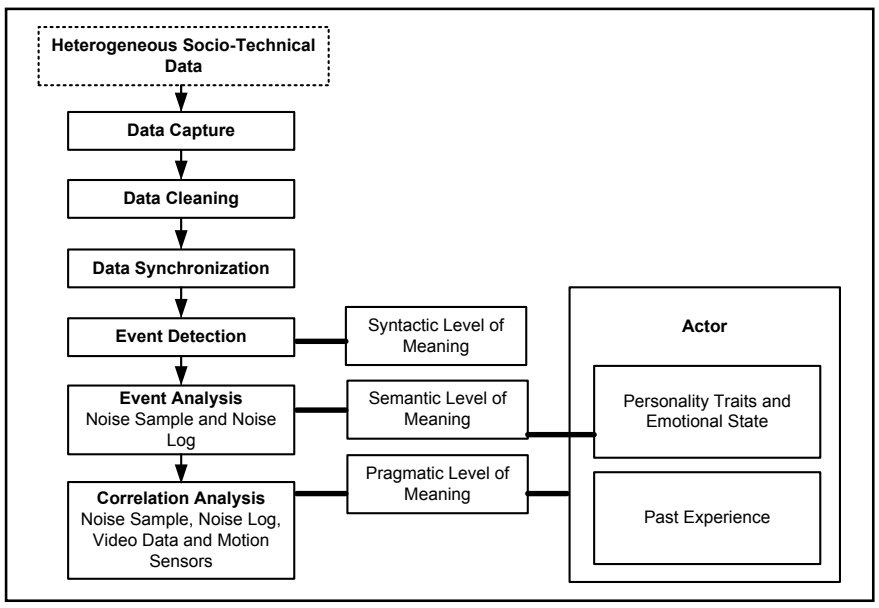

Fig. 2. Socio-Technical Multi-Sensor Architecture

Environmental noise levels are normally measured externally, however, as we are focusing on the impact of nocturnal noise on an actor's sleep patterns, we measured noise from internally located microphones. Three microphones were used: a usb Blue Yeti microphone with a frequency response rate of $20-20,000 \mathrm{~Hz}$ was located centrally in a domestic property. This was supplemented by portable microphones located in the front and back rooms of the property. Passive infra-red motion sensors were located within the property, with further motion sensors linked to external cameras. A total of 14 internal and external video cameras recorded events in the environment in order to help identify potential noise sources.

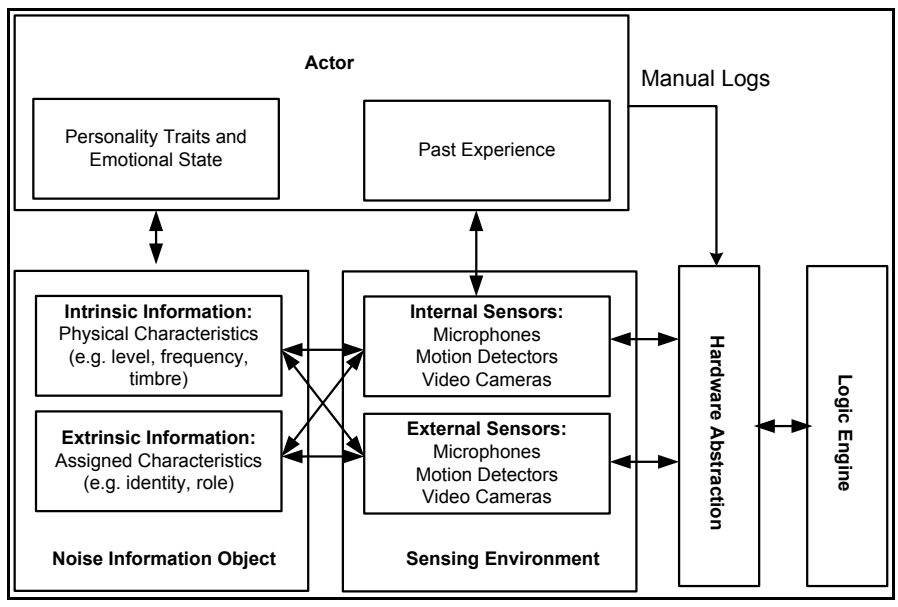

Fig. 3. Data Management Framework

Fig. 3 shows the data management framework used. The data were first cleaned, removing sections contaminated by the experiment set up and shut down. The multiple data streams were synchronized and then Audacity open source software was used to analyse the noise data captured. Timestamped noise samples were identified and compared with the noise logs maintained by the occupants. The noise events were then correlated with data from other sensors to assist in identifying the noise and validating the occupants' interpretation of the noise.

\section{RESULTS AND DISCUSSION}

Over 60TB of data have been captured to date, focusing on the nocturnal period between 22:00 GMT and 08:00 GMT in properties in a urban area of the UK. Samples of noise captured are shown in Table I to provide an indication of the range of noises recorded each night. Previous annotations of the urban soundscape such as [14] identify street noise such as car horns, sirens and children playing. Our dataset includes a wider range of urban noise focusing on unknown night-time sounds and directed disturbances such as stones thrown at windows.

Analysis of the data shows that the syntactic and semantic information provide limited information content about the noise. The pragmatic meaning attributed to the noise object is dependent on the current environment (night time) and the actor's past experience. Actors only react to stimuli that they perceive is useful or relevant to survival [4]. The level, frequency or duration of the noise did not on its own cause concern, for example, loud noise from traffic was disregarded and often not noticed; there was no correlation in noise recording levels and manual noise logs. In contrast, unidentified noises or noises perceived as signalling potential danger or damage to property were more disturbing to occupants.

Actors react to the recognition of the noise, rather than the actual noise [4]. There is a difference between the tangible aspects of the built environment and intangibles that directly influence an actor's experience of the environment [15]. There are also socio-cultural differences in how individuals react to noise [16]. High level noise intensities initiate a 'fight-orflight' response but lower levels of noise attribute to parasympathetic withdrawal [17].

We concur with the experience of [18] that it is difficult to analyse raw environmental audio data captured due to background noise, overlapping sounds and distance variation. Additional sensors are therefore needed to capture data about the environment to assist in verifying the actor's identification of the noise. For example, data captured at 22:52 from motion sensors and video cameras confirmed the occupant's identification of the noise as a door squeaking, and verified that it was caused by a member of the household (Table I). Further work is needed to differentiate noise generated from internal and external sources.

\section{CONCLUSION}

The intrinsic syntactic information of noise objects such as volume can be objectively measured but objective measures do not truly capture or convey the impact of noise in the environment. The lived experience of the noise needs to be considered. A noise phenomenology has been proposed that includes extrinsic information about the identification and context of the noise in order to understand its impact on the actors and their experience of the environmental noise. A multimodal approach to capturing data about the context of the noise is needed that brings together experts in sensor networks, environmental monitoring and information management, adopting a socio-technical approach to examine the lived experience of noise. 
TABLE I. INFORMATION LEVELS OF NOISE

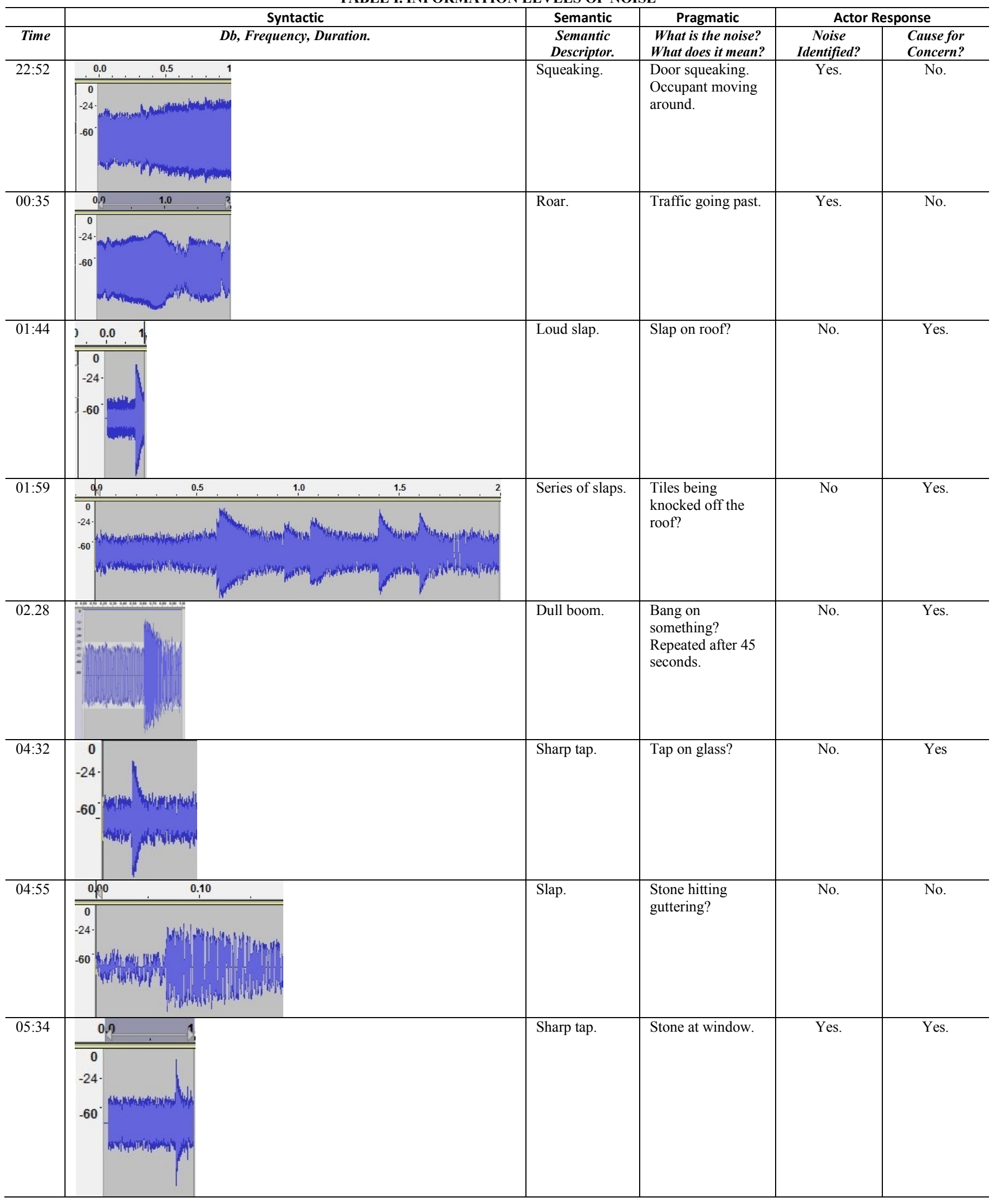




\section{ACKNOWLEDGMENT}

We would like to thank the participants who gave us access to their homes to investigate the nocturnal environment that they endure.

\section{REFERENCES}

[1] D. Halperin, "Environmental noise and sleep disturbances: A threat to health?," Sleep Science, vol. 7, no. 4, pp. 209-212, 2014.

[2] WHO:World Health Organization, "Burden of disease from environmental noise: Quantification of healthy years lost in Europe," [Online], www.euro.who.int/ data/assets/pdf file/0008/136466/e94888.pdf, accessed June 17, 2018.

[3] B. Griefahn and M. Spreng, "Disturbed sleep patterns and limitation of noise," Noise Health, vol. 6, no. 22, pp. 27-33, 2004.

[4] D. Rocchesso, "Phenomenology of sound events," The Sounding Object Report, 2001, funded by the European Community, IST Project No: IST-2000-25287, [Online], www.soundobject.org, accessed June 17, 2018.

[5] T. Gorichanaz and K. F. Latham, "Document phenomenology: A framework for holistic analysis," J. Doc. vol. 72, no. 6, pp. 1114-1133, 2016.

[6] J. Sarraipa, M. Ferro-Beca, C. Marques-Lucena and R. JardimGoncalves, "Knowledge management support in sensing enterprises establishment," Proc. $19^{\text {th }}$ IFAC 2014, Cape Town, South Africa, 24-29 August, 2014.

[7] B. C. O'Connor, J. Kearns, and R. L., Anderson, Doing Things with Information: Beyond Indexing and Abstracting, Libraries Unlimited, Westport, CT, 2008.

[8] M. J. Bates, "Information and knowledge: an evolutionary framework for information science," Information Research, vol. 10, no. 4, paper $239,2005$.
[9] Z. C. Y. Chan, Y-L. Fung and W-T. Chien, "Bracketing in phenomenology: Only undertaken in the data collection and analysis process?,' TQR, vol. 18, no. 59, pp. 1-9, 2013.

[10] K. S. Latham, "Experiencing documents," J. Doc, vol. 70, no. 4, pp. 544-651, 2014

[11] J. Salaman, C. Jacoby and J. P. Bello, "A dataset and taxonomy for urban sound research," Proc. ACMMM 2014, November 3-7, 2014, Orlando.

[12] D. While, J. Krasniewicz and S. Cox, "The development of a unified API for smart home systems.", $1^{\text {st }}$ IOTS, co-located with EuroTRUSTAmi 2008 Conf., September 18-19, 2008, Sophia-Antipolis, French Riviera.

[13] D. While, J. Krasniewicz and S. Cox, "A generic monitoring Framework for pan European energy usage and environmental monitoring," IEEE Comp.Conf., July 18-20, 2017, London, UK.

[14] J. Salamon, D. MacConnel, M. Cartwright, P. Li and J. P. Bello, "SCAPER: a library for soundscape synthesis and augmentation," IEEE WASPAA 2017, October 15-18, 2017, New Paltz, NY, pp. 344-348.

[15] D. Boyd, M. Mayouf and S. Cox, 'Clients' and users' perceptions of BIM: a study in phenomenology,' CIB World Congress, Tampere, Finland, May 30 - June 03, 2016.

[16] P. Lercher, "Environmental noise and health: an integrated research perspective,' Environ. Int., vol. 22, no. 1, pp. 117-129, 1996.

[17] U. Kraus, A. Schneider, S. Breitner, R. Hampel, R. Rückerl, M. Pitz, U. Geruschkat, P, Belcredi, K, Radon and A. Peters, A., "Individual daytime noise exposure during routine activities and heart rate variability in adults: a repeated measures study," Environ. Health Perspect., vol. 121, no. 5, pp. 607-613, 2013.

[18] J. Zhang, K. Huang, M. Cottman-Fields, A. Truskinger, P. Roe, S. Duan, $\mathrm{X}$. Dong, M. Towsey and J. Wimmer, "Managing and analysing big audio data for environmental monitoring,", IEEE 16th ICCSE, Sydney, Australia, December 3-5, 2013, pp. 997-1004. 